\title{
Quantum pump driven fermionic Mach-Zehnder interferometer
}

\author{
S.-W. V. Chung ${ }^{1,2}$, M. Moskalets ${ }^{3}$, P. Samuelsson ${ }^{4}$ \\ ${ }^{1}$ Department of Electronics Engineering and Institute of Electronics, \\ National Chiao-Tung University, Hsinchu 30010, Taiwan \\ ${ }^{2}$ Department of Electrophysics, National Chiao-Tung University, Hsinchu 30010, Taiwan \\ ${ }^{3}$ Department of Metal and Semiconductor Physics, \\ National Technical University "Kharkiv Polytechnic Institute", 61002 Kharkiv, Ukraine \\ ${ }^{4}$ Division of Mathematical Physics, Lund University, Sölvegatan 14 A, S-223 62 Lund, Sweden
}

(Dated: September 7, 2018)

\begin{abstract}
We have investigated the characteristics of the currents in a pump-driven fermionic Mach-Zehnder interferometer. The system is implemented in a conductor in the quantum Hall regime, with the two interferometer arms enclosing an Aharonov-Bohm flux $\Phi$. Two quantum point contacts with transparency modulated periodically in time drive the current and act as beam-splitters. The current has a flux dependent part $I^{(\Phi)}$ as well as a flux independent part $I^{(0)}$. Both current parts show oscillations as a function of frequency on the two scales determined by the lengths of the interferometer arms. In the non-adiabatic, high frequency regime $I^{(\Phi)}$ oscillates with a constant amplitude while the amplitude of the oscillations of $I^{(0)}$ increases linearly with frequency. The flux independent part $I^{(0)}$ is insensitive to temperature while the flux dependent part $I^{(\Phi)}$ is exponentially suppressed with increasing temperature. We also find that for low amplitude, adiabatic pumping rectification effects are absent for semitransparent beam-splitters. Inelastic dephasing is introduced by coupling one of the interferometer arms to a voltage probe. For a long charge relaxation time of the voltage probe, giving a constant probe potential, $I^{(\Phi)}$ and the part of $I^{(0)}$ flowing in the arm connected to the probe are suppressed with increased coupling to the probe. For a short relaxation time, with the potential of the probe adjusting instantaneously to give zero time dependent current at the probe, only $I^{(\Phi)}$ is suppressed by the coupling to the probe.
\end{abstract}

\section{INTRODUCTION}

Phase coherence in solid state conductors is a property of fundamental interest. The prospect of solid state quantum information has also put the focus on possible applications based on phase coherence. With the progress of mesoscopic physics it has become possible to experimentally explore the properties of quantum phase coherence in solid state conductors in a controllable way $\underline{\underline{1}}$ As a prominent example, the fermionic (electronic) analog of the well known bosonic (optical) Mach-Zehnder interferometer(MZI) was recently realized by Ji et $a t^{2}$ and further investigated in Refs. [3,4]. The absence of closed electron orbits makes the MZI the most elementary interferometer and therefore of particular interest.

The MZI experiments $2,3,4$ were all implemented in a conductor in the integer quantum Hall regime, where the electrons propagate along unidirectional, quantum mechanical edge states and quantum point contacts (QPCs) act as beam splitters. In the experiments $2,3,4$ the visibility of the conductance oscillations as a function of flux $\Phi$ were reduced below the ideal value, a signature of dephasing of the electrons propagating along the edges. Dephasing in the MZI was investigated in several theoretical works. Originally, Seelig and Büttiker ${ }^{5}$ investigated the effect of dephasing on the conductance oscillations due to Nyquist noise. Following the experiment in Ref. [2], where also the shot noise was measured, a number of works investigated the effect of dephasing on the current and the noise. The dephasing was introduced via fluctuating classical potentials $6,7,8$ and by coupling the MZI to a quantum bath ${ }^{9}$ as well as to a voltage probe ${ }^{6,7,10}$ Recently these studies were extended to the full distribution of the transferred charge, both for a fluctuating classical potential ${ }^{8}$ as well as a voltage probe $\frac{11,12}{12}$ as a source of dephasing.

Taken together, these theoretical investigations have provided a qualitative picture of the effect of dephasing on transport properties in the MZI. The experimental situation is however not conclusive. In the very recent work by Litvin et $a^{\underline{4}}$ the observed temperature and voltage dependence of the visibility of the conductance oscillations are in good agreement with the noninteracting theory of Ref. [10]. In particular, the effect of the interferometer arm asymmetry is clearly manifested. The overall visibility is however low, a couple of percent. In contrast, in the recent work by Neder et $a^{\underline{3}^{3}}$ the visibility is high, $\sim 60 \%$, but the voltage dependence of the conductance visibility was found to be insensitive to arm asymmetry, however showing a clear lobe structure. A possible explanation for the findings of Ref. [3] was also suggested, invoking interactions between electrons at different edge states. 13 The experimental situation thus motivates further investigations of the coherent transport properties of the MZI.

In this work we propose to investigate the properties of the currents in a pump driven MZI. In contrast to previous work, both experimental and theoretical, all electronic reservoirs are kept at the same potential. The current is instead created via the quantum pump effect, $, 14,15,16,17,18$ by varying periodically the transparencies of the two QPCs. Working in the adiabatic, low pump frequency limit the system is kept close to equi- 
librium. This minimizes the effect of inelastic dephasing and hence allows for a more detailed investigation of the coherence properties.

Theoretically, a large number of investigations of various aspects of quantum pumping have been carried out, a representative collection can be found in Refs. 14, 15, 16, 17, 18, 19, 20, 21, 22, 23, 24, 25, 26, 27, 28, 29, 30, 31 32]. However, only a few experiments aimed at investigating quantum pumping of electrical currents have been performed 33.34 In the MZI the current is a true quantum interference effect. In addition, the elementary structure of the MZI and the fact that the potential applied at the QPC control both the pump effect and the scattering properties of the QPCs makes the MZI a promising candidate for a quantum pump. Previous studies of pumping in mesoscopic interferometers have concerned Aharonov-Bohm, ${ }^{28,35,36}$ double slit-quantum $\operatorname{dot}^{27}$ and two-particle ${ }^{37}$ interferometers, however, to the best of our knowledge, not MZIs.

We use a Floquet scattering approach to the quantum pump problem $38,39,40$ This allows us to calculate the currents in the MZI for arbitrary pumping strength, frequency and temperature. In the Floquet picture, currents arise due to photon assisted interference. It is found that the pumped current contains both an Aharonov Bohm flux dependent part $I^{(\Phi)}$, due to interfering paths enclosing the flux, and a flux independent part $I^{(0)}$. Both current parts depend linearly on the pump frequency in the low frequency, adiabatic regime and show oscillations as a function of frequency in the high frequency, nonadiabatic regime. The oscillations in the non-adiabatic regime occur on two different frequency scales, governed by the interferometer arm length difference and the mean arm length respectively. For the flux dependent current $I^{(\Phi)}$, the oscillations have a constant amplitude while the amplitude of the $I^{(0)}$ oscillations increases linearly with frequency. The two current parts also display a different dependence on temperature; the flux independent part is insensitive to temperature while the flux dependent part is monotonically suppressed with increasing temperature.

In the experiments in Refs. 33, 34 rectification effects made it difficult to distinguish the pumped current. Importantly, in the MZI it is found that in the regime of low amplitude, adiabatic pumping, rectification effects 41 are absent for semitransparent beam-splitters. In order to investigate the effect of dephasing on the pumped current, we consider one of the interferometer arms connected to a voltage probe. Electrons injected into the probe scatter inelastically and hence lose phase coherence before being emitted out of the probe again. Two limiting regimes of the charge relaxation, or $\mathrm{RC}$, time of the voltage probe compared to the pump period are considered; the long relaxation time regime, where the potential of the probe is constant during the measurement, and the short relaxation time regime where the potential of the probe adjusts instantaneously in order to keep zero time dependent current at the probe. In the long time regime the flux dependent current $I^{(\Phi)}$ as well as the part of the flux independent $I^{(0)}$ flowing in the arm connected to the probe are successively suppressed by increasing the dephasing, i.e. the strength of the coupling to the probe. In the short time regime, only $I^{(\Phi)}$ is suppressed by dephasing.

The rest of the paper is organized as follows. In Sec. II the Floquet scattering approach is first presented for an arbitrary mesoscopic scatterer and then applied to the MZI. In Sec. III the properties of the pumped currents are analyzed. Next, in Sec. IV the effects of dephasing are investigated for different response times of the probe. Finally, in Sec. V we conclude.

\section{THEORY AND MODEL}

\section{A. Floquet scattering approach}

For completeness we first briefly review the Floquet scattering approach to pumping in mesoscopic

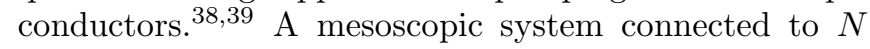
reservoirs via single channel leads is considered. The system is perturbed by some time-dependent parameters which all vary with the same frequency $\omega$. The current flowing in the system in response to the time-periodic perturbation is periodic in time. Expanding the current $I_{\alpha}(t)$ at reservoir $\alpha$ into a Fourier series, we have

$$
\begin{aligned}
I_{\alpha}(t) & =\sum_{l=-\infty}^{\infty} \exp (-i l \omega t) I_{\alpha, l}, \\
I_{\alpha, l} & =\int_{0}^{\mathcal{T}} \frac{d t}{\mathcal{T}} \exp (i l \omega t) I_{\alpha}(t),
\end{aligned}
$$

where $\mathcal{T}=2 \pi / \omega$ is the period of the oscillations. The Fourier component $I_{\alpha, l}$ can be written $\underline{42}$

$$
I_{\alpha, l}=\frac{e}{h} \int_{0}^{\infty} d E\left[\left\langle\hat{b}_{\alpha}^{\dagger}(E) \hat{b}_{\alpha}\left(E_{l}\right)\right\rangle-\left\langle\hat{a}_{\alpha}^{\dagger}(E) \hat{a}_{\alpha}\left(E_{l}\right)\right\rangle\right],
$$

with $\langle\ldots\rangle$ denoting a quantum statistical average. Here $E_{l}=E+l \hbar \omega$ and $\hat{b}_{\alpha}(E)$ and $\hat{a}_{\alpha}(E)$ are annihilation operators for particles coming into and going out from the reservoirs respectively. The operators $\hat{b}_{\alpha}(E)$ and $\hat{a}_{\alpha}(E)$ are related via the Floquet scattering matrix $s_{F}$ as

$$
\hat{b}_{\alpha}(E)=\sum_{\beta=1}^{N} \sum_{n=-\infty}^{\infty} s_{F, \alpha \beta}\left(E, E_{n}\right) \hat{a}_{\beta}\left(E_{n}\right),
$$

where the element $s_{F, \alpha \beta}\left(E, E_{n}\right)$ is the amplitude for scattering of an electron from reservoir $\beta$ at energy $E_{n}$ to reservoir $\alpha$ and energy $E$. All the reservoirs are in thermal equilibrium, giving the average

$$
\left\langle\hat{a}_{\alpha}^{\dagger}\left(E_{n}\right) \hat{a}_{\beta}\left(E_{m}\right)\right\rangle=f_{\alpha}\left(E_{n}\right) \delta_{\alpha \beta} \delta_{n m},
$$

where $f_{\alpha}\left(E_{n}\right)=\left\{1+\exp \left[E_{n} / k_{B} T_{\alpha}\right]\right\}^{-1}$ is the Fermi distribution function with $T_{\alpha}$ the temperature of reservoir $\alpha$ and $k_{B}$ is the Boltzmann constant. 


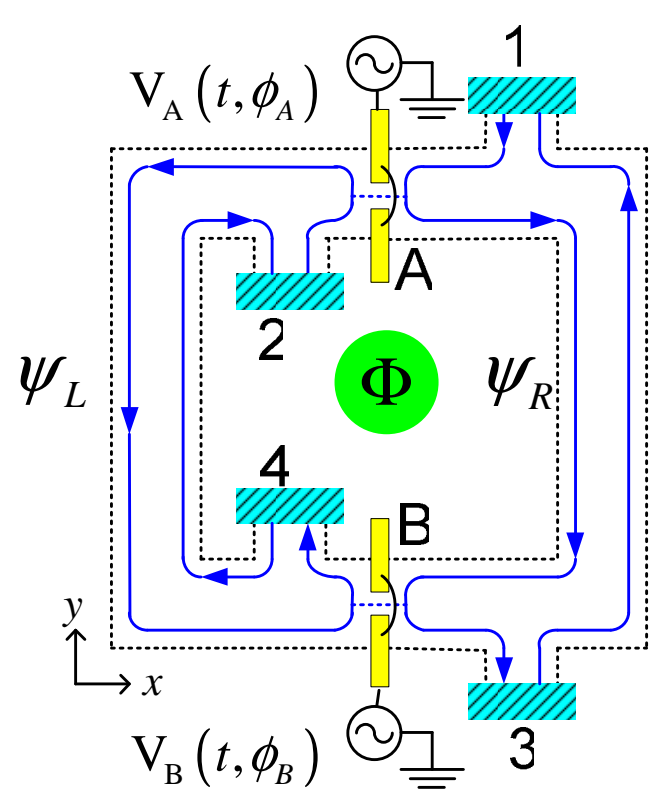

FIG. 1: The pump driven MZI implemented in a conductor in the quantum Hall regime, supporting a single, unidirectional edge state. The conductor is connected to four reservoirs $\alpha=1$ to 4 kept at the same potential. Two electrostatic split gates, at $A$ and $B$, are acting as QPCs. The corresponding gate potentials $V_{A}\left(t, \phi_{A}\right)$ and $V_{B}\left(t, \phi_{B}\right)$, with $\phi_{A}$ and $\phi_{B}$ the pumping phases, vary periodically in time. The time dependent potentials give rise to scattering in both real and energy space and are driving the pump current. An Aharanov-Bohm flux $\Phi$ threads the MZI.

Substituting Eq. (3) into Eq. (2) and taking into account the unitarity of the Floquet scattering matrix,

$$
\sum_{\beta=1}^{N} \sum_{n=-\infty}^{\infty} s_{F, \alpha \beta}^{*}\left(E, E_{n}\right) s_{F, \gamma \beta}\left(E_{l}, E_{n}\right)=\delta_{l, 0} \delta_{\alpha, \gamma},
$$

we can, with Eq. (4), rewrite Eq. (2) as

$$
\begin{aligned}
I_{\alpha, l}= & \frac{e}{h} \int_{-\infty}^{\infty} d E \sum_{\beta=1}^{N} \sum_{n=-\infty}^{\infty}\left[f_{\beta}(E)-f_{\alpha}\left(E_{n}\right)\right] \\
& \times s_{F, \alpha \beta}^{*}\left(E_{n}, E\right) s_{F, \alpha \beta}\left(E_{n+l}, E\right) .
\end{aligned}
$$

Note that to get the above equation we have, compared to Eq. (3), made the shift $E_{n} \rightarrow E$. At $l=0$ the equation (6) defines a dc current

$$
\begin{aligned}
I_{\alpha, d c}= & \frac{e}{h} \int_{-\infty}^{\infty} d E \sum_{\beta=1}^{N} \sum_{n=-\infty}^{\infty}\left|s_{F, \alpha \beta}\left(E_{n}, E\right)\right|^{2} \\
& \times\left[f_{\beta}(E)-f_{\alpha}\left(E_{n}\right)\right] .
\end{aligned}
$$

Through the rest of the paper we will focus on the dccurrent.

\section{B. Mach Zehnder interferometer}

We consider a pump-driven Mach-Zehnder interferometer (MZI) implemented in a conductor in the quantum Hall regime, as shown in Fig. 1. Transport takes place along a single edge state (filling factor one) and is unidirectional. Two electrostatic split gates $A$ and $B$, defining quantum point contacts (QPCs) $j=A, B$, are subjected to time dependent potentials $V_{j}\left(t, \phi_{j}\right)=$ $V_{s j}+V_{j} \cos \left(\omega t+\phi_{j}\right)$ with $\phi_{j}$ the pumping phase. The pumping potentials give rise to scattering of electrons between the edges as well as absorption or emission of one or several quanta of energy $\hbar \omega$. An Aharonov-Bohm flux $\Phi$ penetrates the interior of the interferometer. The conductor is connected to four electronic reservoirs $\alpha=1$ to 4. All four reservoirs are kept at the same potential (grounded) and temperature $T_{\alpha}=T$. Thus, the Fermi distribution functions for all the reservoirs are the same, $f_{\alpha}(E)=f_{0}(E)$, and in the absence of the pumping potentials there is no current flow.

The scattering at the QPCs A and B, taking place both in real space and in energy space, can be described by the Floquet scattering matrices

$$
S_{j}\left(E_{n}, E_{m}\right)=\left(\begin{array}{cc}
r_{j}\left(E_{n}, E_{m}\right) & t_{j}^{\prime}\left(E_{n}, E_{m}\right) \\
t_{j}\left(E_{n}, E_{m}\right) & r_{j}^{\prime}\left(E_{n}, E_{m}\right)
\end{array}\right),
$$

with primed amplitudes for particles incident on the QPCs from left in Fig. 11 The QPCs thus act as inelastic beam splitters. We consider the scattering amplitudes to be independent on energy on the scale of the pump frequency. Consequently, $S_{j}\left(E_{n}, E_{m}\right) \equiv S_{j, n-m}(E)$ can be expressed in terms of the Fourier coefficients for the corresponding frozen scattering matrix ${ }^{43} S_{j}(E, t)$ as

$$
S_{j, n-m}(E)=\int_{0}^{\mathcal{T}} \frac{d t}{\mathcal{T}} e^{i(n-m) \omega t} S_{j}(E, t) .
$$

Moreover, it is assumed that the scale of the energy dependence of the QPC scattering amplitudes is much larger than the thermal energy $k_{B} T$, allowing us to neglect the energy dependence of the Floquet scattering matrix of the QPCs all together, $S_{j, n-m}(E)=S_{j, n-m}$.

Propagating ballistically along the edges between the QPCs the electrons pick up a phase containing both a geometrical part $k_{m} L_{i}$ and a part $\psi_{i}$ due to the AharonovBohm flux, with $i=L, R$. Here $\psi_{L}+\psi_{R}=2 \pi \Phi / \Phi_{0}$ where $\Phi_{0}=h / e$ is the flux quantum. It is assumed that the wavenumber $k_{m}=k\left(E_{m}\right)$ can be taken linear in energy

$$
k_{m} L_{i}=\zeta_{i}(\mu)+\frac{L_{i}}{\hbar v_{D}}(E+m \hbar \omega),
$$

where $\zeta_{i}(\mu)$ is the accumulated phase at the Fermi energy and $v_{D}$ the drift velocity of the edge states. The lengths of the interferometer arms are $L_{L}$ and $L_{R}$ respectively, where we without loss of generality take $L_{L} \geq L_{R}$. The total Floquet scattering amplitude can thus be expressed 
in terms of the scattering amplitudes of the QPCs and the phases acquired along the interferometer arms. For scattering from energy $E$ at reservoir 1 to energy $E_{n}$ at reservoir 3 the amplitude is

$$
\begin{aligned}
s_{F, 31}\left(E_{n}, E\right)= & \sum_{m=-\infty}^{\infty}\left[r_{B, n-m} e^{i k_{m} L_{R}-i \psi_{R}} r_{A, m}\right. \\
& \left.+t_{B, n-m}^{\prime} e^{i k_{m} L_{L}+i \psi_{L}} t_{A, m}\right]
\end{aligned}
$$

and similarly for the other amplitudes. Inserting these scattering amplitudes into Eq. (7) we arrive at the expression for the dc current.

To perform an analysis of the entire parameter space, in the plots we model for simplicity the QPC potentials with oscillating delta function potentials

$$
V_{j}\left(t, \phi_{j}\right)=\delta(x)\left(V_{s j}+2 V_{j} \cos \left(\omega t+\phi_{j}\right)\right) .
$$

We note that this choice leads to completely symmetric scattering matrices, $t_{j, n}=t_{j, n}^{\prime}$ and $r_{j, n}=r_{j, n}^{\prime}$. It is pointed out explicitly in the text below where this additional symmetry qualitatively affects the result. The frozen scattering amplitudes of the QPCs are given by

$$
t_{j}\left(t, \phi_{j}\right)=\frac{1}{1+i m_{e} /\left(\hbar^{2} k_{\mu}\right)\left[V_{s j}+2 V_{j} \cos \left(\omega t+\phi_{j}\right)\right]},
$$

and $r_{j}\left(t, \phi_{j}\right)=t_{j}\left(t, \phi_{j}\right)-1$, with $m_{e}$ the effective electron mass and $k_{\mu}$ the wavenumber at the Fermi energy. This gives from Eq. (9) the Fourier coefficients

$$
\begin{aligned}
t_{j, n} & =\frac{e^{-i n \phi_{j}}}{\sqrt{\left[1+i a_{j}\right]^{2}+b_{j}^{2}}} \\
& \times\left\{\frac{i}{b_{j}}\left[1+i a_{j}-\sqrt{\left(1+i a_{j}\right)^{2}+b_{j}^{2}}\right]\right\}^{|n|}, \\
r_{j, n} & =t_{j, n}-\delta_{n, 0},
\end{aligned}
$$

with $a_{j}=V_{s j} m_{e} /\left(\hbar^{2} k_{\mu}\right)$ and $b_{j}=2 V_{j} m_{e} /\left(\hbar^{2} k_{\mu}\right)$.

\section{PUMPED CURRENT}

In the Floquet scattering picture, the pumping current arises due to interference between different paths of the electrons in energy space, i.e. photon-assisted interference. $\underline{45}$ Due to the absence of closed orbits in the MZI, there are only two different types of interfering paths; the two paths either go along the same interferometer arm, $\mathrm{L}$ or R, or along different arms. The latter paths enclose the flux $\Phi$ in and give rise to an Aharonov-Bohm effect in the pumped current. In Fig. 2 different interfering paths contributing to the current are shown. We note that an Aharonov-Bohm effect in the pumped current was also predicted for other interferometers ${ }^{27,28,35,36}$

It is thus natural to part the total current into a flux dependent and a flux independent part. Focusing on the current at reservoir 3 , we write

$$
I_{3, d c}=I_{3}^{(0)}+I_{3}^{(\Phi)} \text {. }
$$

Inserting the scattering amplitudes in Eq. (11) into the current expression Eq. (77) and carrying out the energy integrals we arrive at the flux independent part

$$
\begin{aligned}
& I_{3}^{(0)}=\frac{e \omega}{2 \pi} \sum_{n=-\infty}^{\infty} \sum_{m=-\infty}^{\infty} \sum_{p=-\infty}^{\infty} n \\
& \times\left\{\left(r_{A, m} r_{A, p}^{*}+t_{A, m}^{\prime} t_{A, p}^{*}\right) t_{B, n-m}^{\prime} t_{B, n-p}^{\prime *}\right. \\
& \times \exp \left[i \omega(m-p)\left(\tau+\frac{\hbar}{2 E_{c}}\right)\right] \\
& +\left(r_{A, m}^{\prime} r_{A, p}^{\prime *}+t_{A, m} t_{A, p}^{*}\right) r_{B, n-m} r_{B, n-p}^{*} \\
& \left.\times \exp \left[i \omega(m-p)\left(\tau-\frac{\hbar}{2 E_{c}}\right)\right]\right\}
\end{aligned}
$$

and the flux dependent part

$$
\begin{aligned}
& I_{3}^{(\Phi)}=\frac{2 e E_{c}}{h} g(T) \sum_{n=-\infty}^{\infty} \sum_{m=-\infty}^{\infty} \sum_{p=-\infty}^{\infty} \sin \left(\frac{n \hbar \omega}{2 E_{c}}\right) \\
& \times 2 \Re\left\{r_{B, n-m} t_{B, n-p}^{\prime *}\left(r_{A, m} t_{A, p}^{*}+t_{A, m}^{\prime} r_{A, p}^{*}\right)\right. \\
& \left.\times \exp \left[i\left(\psi_{L R}+\omega\left[(m-p) \tau+(n-p-m) \frac{\hbar}{2 E_{c}}\right]\right)\right]\right\},
\end{aligned}
$$

where

$$
g(T)=\frac{\pi k_{B} T}{E_{c}} \operatorname{csch}\left(\frac{\pi k_{B} T}{E_{c}}\right),
$$

the phase $\psi_{L R}=\zeta_{L}(\mu)-\zeta_{R}(\mu)-2 \pi \Phi / \Phi_{0}$ and $\Re$ denoting the real part.

In order to explicitly display the relevant energy and time scales we have introduced the asymmetry energy $E_{c}=\hbar v_{D} /\left(L_{L}-L_{R}\right)$ and the average time $\tau=\left(L_{L}+\right.$ $\left.L_{R}\right) /\left(2 v_{D}\right)$ for ballistic propagation between the QPCs. By definition $\tau>\hbar /\left(2 E_{c}\right)$. There are thus three different, possible pumping regimes depending on the relation between the pump frequency $\omega$ and the frequency scales $E_{c} / \hbar$ and $1 / \tau$ : (i) For $\omega \ll 1 / \tau$ the pumping is adiabatic, the total scattering amplitudes of the MZI are independent on energy on the scale of the pumping frequency $\omega$. For non-adiabatic pumping there are in addition two regimes. (ii) In the intermediate frequency regime $1 / \tau \ll \omega \ll E_{c} / \hbar$ the pumped current is independent on the interferometer asymmetry but depends on the total time $\tau$. (iii) For $E_{c} / \hbar \ll \omega$, in the high frequency regime, the pumped current depends both on the asymmetry and the total time.

Several important observations can be made directly from the formal expressions in Eqs. (16) and (17). First, the flux independent current $I_{3}^{(0)}$ is an incoherent sum of the currents pumped through the left and right arms. The two currents are denoted $I_{3 L}^{(0)}$ [upper term in Eq. (16)] and $I_{3 R}^{(0)}$ (lower term) respectively. Each current term, $I_{3 L}^{(0)}$ or $I_{3 R}^{(0)}$, depends explicitly on the time for ballistic propagation through the corresponding left or right arm, $\tau+\hbar /\left(2 E_{c}\right)=L_{L} / v_{D}$ and $\tau-\hbar /\left(2 E_{c}\right)=L_{R} / v_{D}$. 

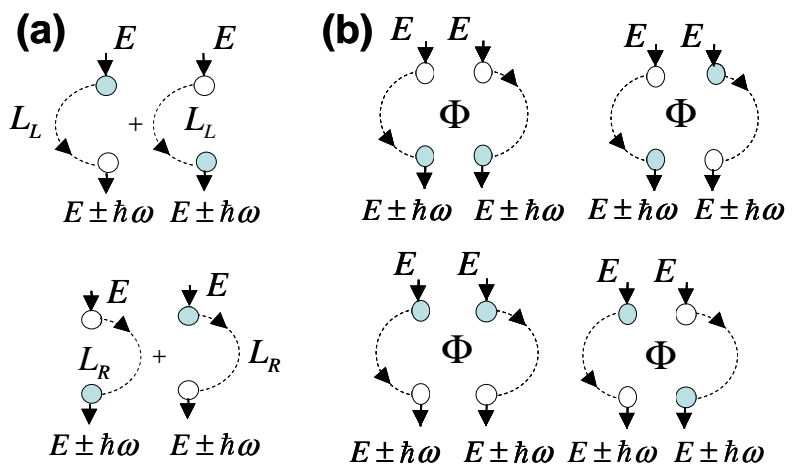

FIG. 2: Two qualitatively different types of first order photonassisted interference processes contributing to the current: (a) along the same spatial paths $\mathrm{L}$ or $\mathrm{R}$ and (b) along the different spatial paths $\mathrm{L}$ and $\mathrm{R}$. The paths in (b) are sensitive to the enclosed flux $\Phi$. Filled balls indicate inelastic scattering, the electrons pick up or lose one quantum of energy $\hbar \omega$, while empty balls indicate elastic scattering.

For the flux dependent current, no such partitioning into left and right arm currents is possible.

Second, while the flux independent current $I_{3}^{(0)}$ is independent on the temperature, the flux dependent current $I_{3}^{(\Phi)}$ is monotonically suppressed with increasing temperature. Despite the fact that both terms are of interference nature, they thus depend on temperature in very different ways. The energy scale of the decay of $I_{3}^{(\Phi)}$ is set by the asymmetry energy $E_{c}$ : the factor $g(T)$ is equal to unity for $k_{B} T \ll E_{c}$ and decays as $\exp \left(-\pi k_{B} T / E_{c}\right)$ for $k_{B} T \gg E_{c}$. This is qualitatively similar to the voltage biased MZI 10 and can be understood as an effect of energy averaging. Notably, the temperature dependence of the current is affected neither by the pumping frequency $\omega$ nor by the average time $\tau$.

Third, the qualitative behavior of the currents as a function of frequency can also be understood from Eqs. (16) and (17). It is clear that both currents $I_{3}^{(0)}$ and $I_{3}^{(\Phi)}$ show oscillations in the non-adiabatic regime as a function of $\omega$, on the scales $E_{c} / \hbar$ and $1 / \tau$. In the intermediate frequency regime (ii), for $1 / \tau \ll \omega \ll E_{c} / \hbar$, the pumped current is however insensitive to the asymmetry and shows oscillations with the basic period $\tau$ only. In regime (iii), for $E_{c} / \hbar \ll \omega$, the pumped current shows oscillations as a function of frequency on both the scales $1 / \tau$ and $E_{c} / \hbar$. For a small asymmetry, $E_{c} \gg \hbar / \tau$, the oscillations show a beating pattern with rapid oscillations on the scale $1 / \tau$ periodically modulated in amplitude on the scale $E_{c} / \hbar$. This is illustrated in the plots in Fig. 3. We point out that for the flux independent current $I_{3}^{(0)}$ the beating pattern can simply be understood as the effect of adding the two currents terms $I_{3 L}^{(0)}$ and $I_{3 R}^{(0)}$ with the two different time periods $\tau \pm \hbar /\left(2 E_{c}\right)$.

Importantly, the amplitudes of the oscillations of $I_{3}^{(0)}$

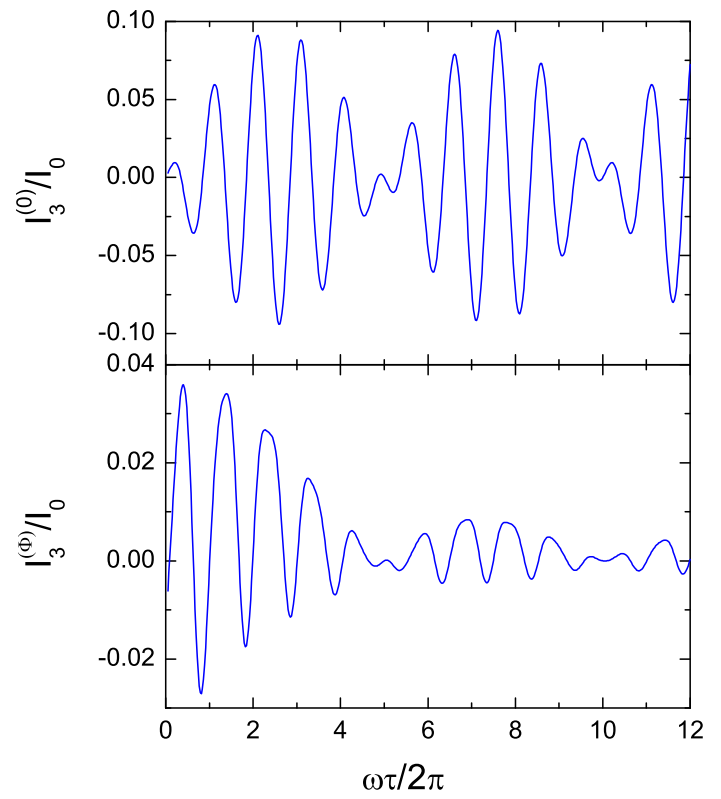

FIG. 3: The flux independent current $I_{3}^{(0)}$ (upper panel) and the flux dependent current $I_{3}^{(\Phi)}$ (lower panel) as a function of pump frequency. Guided by the experiments in Ref. 3. 4 we have taken an asymmetry, $\tau=5 \hbar / E_{c}$, and symmetric static beam splitters, $a_{A}=a_{B}=1$. The other parameters are $b_{A}=0.4, b_{B}=1.3$ (strong pumping), $\phi_{A}=0, \phi_{B}=0.8 \pi$ and $\psi_{L R}=0.8 \pi$.

and $I_{3}^{(\Phi)}$ show a different frequency dependence. As is clear from Eq. (16), in the high frequency regime, $E_{c} / \hbar \ll \omega$, the flux independent current is proportional to $\omega$ while the flux dependent current has no frequency dependent prefactor. Plotting the currents divided by the elementary pumped current $I_{0}=e \omega / 2 \pi$, the oscillations of the normalized current $I_{3}^{(0)} / I_{0}$ have a constant amplitude as a function of frequency while the amplitude of $I_{3}^{(\Phi)} / I_{0}$ decreases as $1 / \omega$. This is illustrated in Fig. 3.

In the adiabatic regime, $\omega \ll 1 / \tau$, both currents $I_{3}^{(0)}$ and $I_{3}^{(\Phi)}$ in general show a linear dependence in $\omega$. We however note that the our choice of a spatially symmetric model potential for the QPCs leads to a flux independent part of the current proportional to $\omega^{2}$. This quadratic frequency dependence is not clearly visible in the plot in Fig. 3. This sensitivity of $I_{3}^{(0)}$ to spatial symmetry is further discussed below.

\section{A. Weak amplitude pumping}

Several of the properties of the pumped current become more transparent in the limit of weak pumping, where only one quantum of energy $\hbar \omega$ can be absorbed or emitted when scattering through the MZI. This allows 
us to write the two current parts on the form

$$
\begin{aligned}
& I_{3}^{(0)}=\frac{-2 e \omega}{\pi} \Im\left\{t_{B}^{\prime} \delta t_{B}^{*^{\prime}}\left(\delta t_{A} t_{A}^{*}+\delta r_{A}^{\prime} r_{A}^{\prime *}\right)\right. \\
\times & \sin \left(\phi_{A}-\phi_{B}-\omega\left[\tau+\frac{\hbar}{2 E_{c}}\right]\right) \\
+ & r_{B} \delta r_{B}^{*}\left(\delta r_{A} r_{A}^{*}+\delta t_{A}^{\prime} t_{A}^{\prime *}\right) \\
\times & \left.\sin \left(\phi_{A}-\phi_{B}-\omega\left[\tau-\frac{\hbar}{2 E_{c}}\right]\right)\right\}
\end{aligned}
$$

with $\Im$ the imaginary part and

$$
\begin{aligned}
& I_{3}^{(\Phi)}=\frac{8 e E_{c}}{h} g(T) \sin \left(\frac{\hbar \omega}{2 E_{c}}\right) \Im\left\{e^{i \psi_{L R}}\right. \\
& \times\left[\left(\delta r_{B} t_{B}^{\prime *}+r_{B} \delta t_{B}^{\prime *}\right)\left(r_{A} \delta t_{A}^{*}+\delta t_{A}^{\prime} r_{A}^{* *}\right)\right. \\
& \times \sin \left(\phi_{A}-\phi_{B}-\omega \tau\right) \\
& \left.\left.+r_{B} t_{B}^{\prime *}\left[\delta r_{A} \delta t_{A}^{*}+\delta t_{A}^{\prime} \delta r_{A}^{\prime *}\right] \sin \left(\frac{\hbar \omega}{2 E_{c}}\right)\right]\right\} .
\end{aligned}
$$

Here we introduced the notation $r_{j, 0}=r_{j}, r_{j, 0}^{\prime}=$ $r_{j}^{\prime}, t_{j, 0}=t_{j}$ and $t_{j, 0}^{\prime}=t_{j}^{\prime}$ for the amplitudes to scatter elastically, without absorbing or emitting any energy quantum, and $r_{j, \pm 1}=\delta r_{j} e^{\mp i \phi_{j}}, r_{j, \pm 1}^{\prime}=\delta r_{j}^{\prime} e^{\mp i \phi_{j}}, t_{j, \pm 1}=$ $\delta t_{j} e^{\mp i \phi_{j}}$ and $t_{j, \pm 1}^{\prime}=\delta t_{j}^{\prime} e^{\mp i \phi_{j}}$ for the amplitudes to emit $(-)$ or absorb $(+)$ a single energy quantum.

Importantly, the terms in the current expressions directly correspond to the first order scattering processes shown in Fig. 2. For $I_{3}^{(0)}$, the upper term in Eq. (19), $I_{3 L}^{(0)}$, arises due to interference between electrons that propagate along the left arm and pick up or lose a quantum $\hbar \omega$ at either $A$ or $B$. These processes are shown at the top of panel (a) in Fig. 2. The lower term in Eq. (19), $I_{3 R}^{(0)}$, arises from the corresponding processes for electrons propagating in the right interferometer arm.

For the flux dependent current $I_{3}^{(\Phi)}$, the interfering paths go along different interferometer arms $L$ and $R$. The upper term in Eq. (20) arises from processes where electrons pick up or lose one quantum $\hbar \omega$ at different QPCs $A$ and $B$. These processes are depicted to the right in panel (b). The lower term in Eq. (20) arises from processes where both electrons pick up or lose one quantum $\hbar \omega$ at the same QPC, $A$ or $B$, depicted to the left in panel (b). Importantly, electrons which scatter inelastically at the same QPC pick up the same information on the pumping phase. Consequently, the corresponding interference term is independent on the pumping phase, as seen in the lower term in Eq. (20).

The weak amplitude expressions for the current also clearly demonstrate the origin of the sign change of the current as a function of frequency, as shown in Fig. 3 . In the low frequency, adiabatic limit the weak amplitude pumped current is always 17 proportional to $\sin \left(\phi_{A}-\phi_{B}\right)$, i.e. the sign of the current is determined by the pumping phase difference. In Eqs. (19) and (20) the frequency formally enters the current expressions as an additional pumping phase, thus leading to an oscillating sign of the current as a function of frequency.

\section{B. Adiabatic, weak pumping}

It is of particular importance to consider the weakly pumped currents in the adiabatic limit, where the effects of inelastic dephasing are minimized. In the adiabatic limit the current reduces to, using the unitarity relations in Eq. (5)

$$
\begin{aligned}
I_{3, a d}^{(0)} & =\frac{i e \omega}{\pi} \sin \left(\phi_{A}-\phi_{B}\right)\left(r_{B} \delta r_{B}^{*}+r_{B}^{*} \delta r_{B}\right) \\
& \times\left(\delta t_{A} t_{A}^{*}-\delta t_{A}^{\prime} t_{A}^{*}-\delta r_{A} r_{A}^{*}+\delta r_{A}^{\prime} r_{A}^{*}\right)
\end{aligned}
$$

and

$$
\begin{aligned}
I_{3, a d}^{(\Phi)} & =\frac{2 e \omega}{\pi} \sin \left(\phi_{A}-\phi_{B}\right) g(T) \Im\left\{e^{i \psi_{L R}}\right. \\
& \left.\times\left(\delta r_{B} t_{B}^{* *}+r_{B} \delta t_{B}^{*}\right)\left(r_{A} \delta t_{A}^{*}+\delta t_{A}^{\prime} r_{A}^{* *}\right)\right\}
\end{aligned}
$$

Note that the second line in Eq. (21) is purely imaginary. From the expression of the flux independent current $I_{3, a d}^{(0)}$ we see explicitly the dependence on spatial symmetry of QPC $A$. For a completely symmetric scattering potential, i.e. primed scattering amplitudes equal to unprimed, the adiabatic phase independent current is zero and the low frequency current is $\propto \omega^{2}$. We point out that the absence of a noticeable magnetic flux through the point contact area, i.e. $t_{A}=t_{A}^{\prime}$, is not enough to suppress the adiabatic current. We also note that only the spatial symmetry of QPC $A$ is relevant, a consequence of the chiral transport. That is, reversing the sign of the quantum Hall magnetic field, the pumped currents, now at reservoirs 1 and 2, would be sensitive to the symmetry of QPC $B$ only.

From the dependence of $I_{3, a d}^{(\Phi)}$ on $\psi_{L R}$, as well as the fact that $\psi_{L R}$ depends both on the Aharanov-Bohm flux as well as phases picked up propagating along the edges [see definition below Eq. (18)], we can conclude that the flux dependent part has no definite magnetic field symmetry. We also note that in the adiabatic expression for the flux dependent current, the lower term in Eq. (20), independent on the pumping phases, does not contribute.

\section{Rectification effects}

For mesoscopic conductors, an unavoidable feature is stray capacitances between the various circuit elements, i.e. the electronic reservoirs, the electrostatic gates and the mesoscopic sample itself. A capacitive coupling between the pumped QPC gates and the electronic reservoirs induces an ac potential at the reservoirs (for nonzero impedance of the current measurement circuit). This gives rise to a rectification current which can obscure the pumped current, 26,33,34,39,41 In the MZI, for weak, adiabatic pumping, the rectified dc-current is in the most general situation given by 41

$$
\begin{aligned}
I_{3, \text { rect }} & =c_{A 1} \frac{\partial G_{31}}{\partial V_{A}}+c_{B 1} \frac{\partial G_{31}}{\partial V_{B}} \\
& +c_{A 2} \frac{\partial G_{32}}{\partial V_{A}}+c_{B 2} \frac{\partial G_{32}}{\partial V_{B}}
\end{aligned}
$$


where the constants $c_{j \alpha}$ depend on the capacitive couplings, the impedance of the measurement circuit, the pumping phases and the pumping amplitudes and $G_{\alpha \beta}=$ $d I_{\alpha} / d V_{\beta}$ is the conductance. From the theory for the conductance of the MZI in Ref. [10] we have

$$
\begin{aligned}
\frac{\partial G_{31}}{\partial V_{A}} & =\frac{\partial T_{A}}{\partial V_{A}} \frac{\partial G_{31}}{\partial T_{A}}=\frac{\partial T_{A}}{\partial V_{A}} \frac{2 e^{2}}{h} \\
& \times\left(T_{B}-R_{B}+H \frac{R_{B} T_{B}\left(R_{A}-T_{A}\right)}{2 \sqrt{R_{A} T_{A} R_{B} T_{B}}}\right)
\end{aligned}
$$

and similarly for the other conductance derivatives. Here $T_{A}=1-R_{A}=\left|t_{A}\right|^{2}=\left|t_{A}^{\prime}\right|^{2}$ is the transmission probability of the static QPC $A$ and $H=H\left(k_{B} T, E_{c}, \Phi\right)$ a function dependent on the different energy scales $k_{B} T$ and $E_{c}$ and the enclosed flux $\Phi$. The rectification current and the pumped current thus depend differently on the scattering parameters, the magnetic flux and the energy scales, allowing one to distinguish experimentally between the two currents. In particular, from Eq. (24) it is clear that working with semitransparent beam splitters $T_{A}=R_{A}=1 / 2$ and $T_{B}=R_{B}=1 / 2$ the rectification currents are zero. This holds independently on the values of the individual couplings $c_{j \alpha}$.

We also emphasize that induced ac-potentials at the reservoirs do not simply lead to a rectification current which is incoherently added to the pumped current; there is in general also a current due to interference between processes responsible for the pumped current and the rectification current $\underline{\underline{39}}$ However, the induced ac-potential is proportional to $\underline{41} d V_{A} / d t, d V_{B} / d t \sim \omega$ and in the weak amplitude, adiabatic limit the interference current is consequently $\propto \omega^{2}$.

A related issue is the effect of the temporary charging of the MZI itself due to the pumping. In the calculations and discussions of the pumped current above we have neglected this effect, i.e we have considered noninteracting electrons. An interacting theory should also take into account screening at the edges and the effect of capacitive couplings of the edges to e.g. each other and to the electrostatic gates. This would require a self-consistent determination of the time dependent edge state potentials $\stackrel{46,47}{=}$ Such an interacting theory however goes beyond the scope of this paper.

\section{DEPHASING}

An important problem in the study of interference phenomena in mesoscopic conductors is decoherence. Phase information of the electrons propagating in the MZI is lost. Various approaches to dephasing in the voltage biased MZI were discussed in the introduction. Here we introduce dephasing in the MZI by coupling one of the arms of the interferometer to a voltage probe as shown in Fig. 4. A voltage probe is an additional electronic reservoir with the potential left floating. Electrons entering the voltage probe are incoherently fed back into the interferometer arm, thereby suppressing phase coherence.
Voltage probes as means to introduce incoherent, inelastic scattering was proposed by Büttiker $\stackrel{48,49}{\text { The concept }}$ has thereafter been extended and applied to a large number of mesoscopic conductors, both theoretically and experimentally. A recent account of this development was given in Ref. [50].

For our purposes, in quantum Hall systems the theory of current and noise in the presence of voltage probes was developed in Ref. 51] and applied to a voltage biased MZI in Refs. [6, 7, 10]. We point out that the very recent experiments by Oberholzer et al, $\stackrel{52}{\text { investigating }}$ the current cross correlations in a quantum Hall geometry coupled to a voltage probe, were in excellent agreement with the theory of Ref. [51]. Moreover, dephasing of the pumped current via voltage probes was considered in Refs. [53, 54, 55].

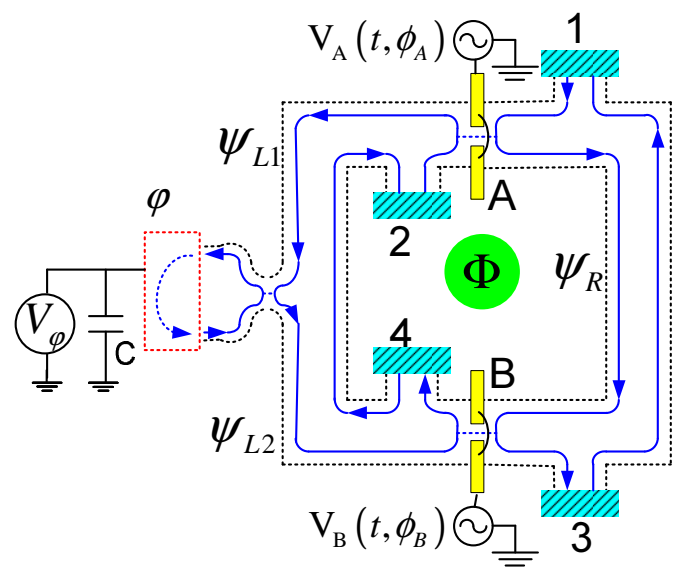

FIG. 4: The pump driven MZI of Fig. 1 with the left arm connected with strength $\epsilon$ to a voltage probe $\varphi$. The dynamics of the potential $V_{\varphi}$ of the probe is governed by the charge relaxation time (see text).

The elastic scattering matrix of the contact to the probe is given by

$$
\left(\begin{array}{cc}
\sqrt{1-\epsilon} & i \sqrt{\epsilon} \\
i \sqrt{\epsilon} & \sqrt{1-\epsilon}
\end{array}\right)
$$

Here $\epsilon$ governs the strength of the dephasing. For $\epsilon=1$ the dephasing is complete, i.e. the probe is fully coupled to the MZI and all electrons propagating along the interferometer arm enter the probe. For $\epsilon=0$ the transport is fully coherent, the probe is decoupled from the MZI.

An important property of the voltage probe is the charge relaxation time of the voltage probe $\frac{11,12}{1}$ i.e. the time scale on which the probe is charged or discharged. The charge relaxation time determines the dynamics of the potential $V_{\varphi}(t)$ of the probe and consequently the response to the injected, time dependent charge. The charge relaxation time is given by the RCtime $\tau_{R C}=R C$, with $R$ the charge relaxation resistance and $C$ the capacitance (see Fig. (4). Büttiker and one 
of the authors 53 considered adiabatic pumping in a conductor connected to a voltage probe, assuming instantaneous charge conservation at the probe, i.e. a relaxation time much shorter than the pump period. Cremers and Brouwer $^{54}$ investigated the pumped current in a chaotic quantum dot in the same short relaxation time limit. Considering the experimental setup of Ref. 33], Polianski and Brouwer ${ }^{56}$ investigated the adiabatic dynamics of the floating potential of reservoirs. They considered the two limiting cases of long and short relaxation time compared to the pump period. Here we will consider the same limiting cases of short and long relaxation time for the voltage probe, without the restriction to adiabatic pumping.

\section{A. Long charge relaxation time $\tau_{R C} \gg \mathcal{T}$}

First, the case with long relaxation time is considered, where the potential of the probe does not react on the injected charge on the time scale of the pumping period, $\tau_{R C} \gg \mathcal{T}$. In this situation the potential of the probe is constant during the measurement. Since the particles entering the probe have scattered at the adiabatically pumped QPC A only, there is no dc-current flow into the probe and the potential of the probe $V_{\varphi}$ stays at the same potential as the four reservoirs of the MZI. We thus have an extended pumping problem with five instead of four equipotential reservoirs, which can be treated along the same lines as above.

First, the coupling of the MZI to the probe leads to a modification of the scattering amplitudes in Eq. (11), as $s_{F, \alpha \beta} \rightarrow \tilde{s}_{F, \alpha \beta}$, with e.g.

$$
\begin{aligned}
& \tilde{s}_{F, 31}\left(E_{n}, E\right)=\sum_{m=-\infty}^{\infty}\left[r_{B, n-m} e^{i k_{m} L_{R}-i \psi_{R}} r_{A, m}\right. \\
& \left.+\sqrt{1-\epsilon} t_{B, n-m}^{\prime} e^{i k_{m} L_{L}+i \psi_{L}} t_{A, m}\right]
\end{aligned}
$$

and similar for the other amplitudes to scatter from reservoirs 1 and 2 to 3 and 4 . Moreover, there are now the amplitudes to scatter to and from the probe, as e.g. from $\varphi$ to 3

$$
\tilde{s}_{F, 3 \varphi}\left(E_{n}, E\right)=i \sqrt{\epsilon} t_{B, n}^{\prime} e^{i k_{0} L_{L 2}},
$$

where $L_{L 2}$ is the length along the left edge between the probe and QPC B (see Fig. 4). Inserting the scattering amplitudes $\tilde{s}_{F, \alpha \beta}$ into the formula for the dc-current, Eq. (77), we arrive at the result that the coherent current is modified as

$$
\begin{aligned}
I_{3 L}^{(0)} & \rightarrow(1-\epsilon) I_{3 L}^{(0)} \\
I_{3}^{(\Phi)} & \rightarrow \sqrt{1-\epsilon} I_{3}^{(\Phi)}
\end{aligned}
$$

The flux independent current in the arm to which the probe is coupled, $I_{3 L}^{(0)}$, is successively suppressed for increasing coupling $\epsilon$ to the probe. For perfect coupling, $\epsilon=1$, the current $I_{3 L}^{(0)}$ is zero. The pumped current flowing through the arm not connected to the probe is however unaffected by the coupling to the probe. In contrast, the entire flux independent current $I_{3}^{(\Phi)}$ is suppressed for increasing coupling to the probe, down to zero for perfect coupling.

\section{B. Short charge relaxation time $\tau_{R C} \ll \mathcal{T}$}

In the limit of a response time much shorter than the pumping period, $\tau_{R C} \ll \mathcal{T}$, the potential of the probe $V_{\varphi}(t)$ adjusts instantaneously, in order to keep the time dependent current at the probe zero, $I_{\varphi}(t)=0$. This corresponds to that all frequency components of the current [see Eq. (1)] are zero,

$$
I_{\varphi, l}=0
$$

Since the electrons entering the voltage probe are rapidly thermalized, the electrons in the probe can be considered in the same way as electrons in a reservoir with oscillating potential, i.e. in dynamical equilibrium. The oscillating potential gives rise to a nonequilibrium distribution of the electrons leaving the probe. Formally, assuming a uniform potential of the probe, we follow the scattering approach in Ref. [47] and introduce annihilation operators for the electrons emitted from the probe as

$$
\hat{a}_{\varphi}^{\prime}(E)=\sum_{n=-\infty}^{\infty} L_{-n} \hat{a}_{\varphi}\left(E_{n}\right)
$$

Here the operators $\hat{a}_{\varphi}(E)$ describe equilibrium electrons: $\left\langle\hat{a}_{\varphi}^{\dagger}\left(E_{n}\right) \hat{a}_{\varphi}\left(E_{m}\right)\right\rangle=f_{0}\left(E_{n}\right) \delta_{n, m}$. The amplitudes $L_{n}$ are defined as

$$
L_{n}=\int_{0}^{\mathcal{T}} \frac{d t}{\mathcal{T}} \exp (i n \omega t) \exp \left(-i \int d t e V_{\varphi}(t) / \hbar\right)
$$

The annihilation operators for particles injected into the probe can then be written as

$$
\begin{aligned}
& \hat{b}_{\varphi}(E)=\sum_{n=-\infty}^{\infty}\left[s_{F, \varphi 1}\left(E, E_{n}\right) \hat{a}_{1}\left(E_{n}\right)\right. \\
& \left.+s_{F, \varphi 2}\left(E, E_{n}\right) \hat{a}_{2}\left(E_{n}\right)+\sqrt{1-\epsilon} L_{-n} \hat{a}_{\varphi}\left(E_{n}\right)\right] .
\end{aligned}
$$

Importantly, the amplitudes $L_{n}$ in Eq. (30) effectively describe forward, inelastic scattering from the probe out into the MZI. We can consequently combine the amplitudes $L_{n}$ for excitation of the electrons in the probe and the amplitudes $\tilde{s}_{F, \alpha \beta}$ for scattering in the MZI with zero probe potential into a new, unitary Floquet scattering matrix $\bar{s}_{F, \alpha \beta}$. This gives $\bar{s}_{F, \alpha \beta}\left(E, E_{n}\right)=\tilde{s}_{F, \alpha \beta}\left(E, E_{n}\right)$ for $\beta \neq \varphi$ and $\bar{s}_{F, \varphi \varphi}\left(E, E_{n}\right)=\sqrt{1-\epsilon} L_{-n}$ and similarly for $\bar{s}_{F, 3 \varphi}$ and $\bar{s}_{F, 4 \varphi}$. It is then possible to proceed as above and insert the scattering amplitudes $\bar{s}_{F, \alpha \beta}$ into the formula for the fourier components of the current, Eq. (11). 
This gives

$$
\begin{aligned}
I_{\varphi, l}= & \frac{e}{h} \int_{0}^{\infty} d E \sum_{m=-\infty}^{\infty}\left[f_{0}\left(E_{m}\right)-f_{0}(E)\right] \\
& \times \sum_{\beta} \bar{s}_{F, \varphi \beta}^{*}\left(E, E_{m}\right) \bar{s}_{F, \varphi \beta}\left(E_{l}, E_{m}\right),
\end{aligned}
$$

where $\beta$ runs over 1,2 and $\varphi$. The requirement of instantaneous current conservation, Eq. (29), then directly gives

$$
\sum_{\beta} \bar{s}_{F, \varphi \beta}^{*}\left(E, E_{m}\right) \bar{s}_{F, \varphi \beta}\left(E_{l}, E_{m}\right)=0,
$$

which in terms of the amplitudes $L_{n}$ can be written

$$
L_{m}^{*} L_{m+l}=\frac{1}{\epsilon} \sum_{\beta=1,2} \bar{s}_{F, \varphi \beta}^{*}\left(E, E_{-m}\right) \bar{s}_{F, \varphi \beta}\left(E_{l}, E_{-m}\right) .
$$

We will then use Eq. (35) to calculate the dc-current at reservoir 3. The dc-current is given by Eq. (7), now with the scattering amplitudes $\bar{s}_{F, 3 \beta}$. Via the amplitude $\bar{s}_{F, 3 \varphi}$ the current depends on the product $L_{m}^{*} L_{m+l}$. Inserting the expression for $L_{m}^{*} L_{m+l}$ from Eq. (35) we arrive at the result for the flux dependent part of the current

$$
I_{3}^{(\Phi)} \rightarrow \sqrt{1-\epsilon} I_{3}^{(\Phi)}
$$

while in contrast to the long relaxation time result in Eq. (28), the current part $I_{3}^{(0)}$ is unaffected by the dephasing.

Importantly, the different dephasing behaviors in the two regimes of probe relaxation time are clearly manifested in the pumped current. In the long time regime the suppression of the current in the left arm $I_{3 L}^{(0)}$ leads to that the measured current only depends on the time for ballistic propagation in the right arm, see Eq. (16). As a consequence, the beating pattern in the frequency dependence of the pumped current (see Fig. 3) is suppressed on increasing dephasing. In the short time regime there is no such suppression.

\section{CONCLUSIONS}

We have investigated the pumped currents in a MZI implemented in a conductor in the quantum Hall regime. The motivation for our investigation was twofold. First, a MZI is the most elementary interferometer, due to the absence of closed electronic orbits. In our proposal the pumped current in the MZI is moreover operated solely by modulating the potential at the two QPCs. This makes pumping in the MZI both fundamentally important and experimentally achievable. Second, recent experiments 2.3 .4 on transport in a voltage biased MZI has demonstrated the relevance of dephasing and raised a number of questions on the coherence properties of MZIs. Working in the adiabatic pumping regime makes it possible to investigate these coherence properties close to equilibrium, keeping dephasing at a minimum.

The dependence of the current on pumping frequency, pumping strength, temperature and lengths of the arms of the MZI were investigated. The two parts of the current, the flux dependent and the flux independent ones, were demonstrated to depend in a qualitatively different way on frequency and temperature. The two current parts also showed a different sensitivity to dephasing, introduced by coupling a voltage probe to one of the interferometer arms. The flux dependent current was successively suppressed for increasing coupling, while only the part of the flux independent current flowing in the arm connected to the probe was sensitive to dephasing in the limit of long charge relaxation time of the probe. We also demonstrated that rectification effects, preventing an unambiguous demonstration of quantum pumping of current, are absent in the MZI when working with semitransparent beam splitters in the adiabatic, weak pumping regime.

In a broader perspective, a better understanding and control of coherence properties of edge state transport is important for a successful realization of two-particle Hanbury Brown Twiss interferometers,,$\underline{57}$ entanglement production ${ }^{57,58}$ and quantum state transfer ${ }^{59}$ in quantum Hall systems. In the context of entanglement, an unambiguous demonstration of quantum pumping in the MZI also opens up for schemes for entanglement generation based on quantum pump effects $\stackrel{37,60,61,62,63}{2}$

\section{ACKNOWLEDGEMENTS}

The authors would like to thank M. Büttiker for suggesting the problem and providing important comments on the work. We also thank M. Polianski for a critical reading of the manuscript. S.-W.V.C. also thanks C.S. Tang and C.S. Chu for comments. S.-W.V.C. is supported by the National Science Council of Taiwan under the Grant Nos. NSC94-2112-M-009-017, MOE ATU Program, and NSC 93-2119-M-007-002 (NCTS). M.M. appreciates the support of the National Center for Theoretical Sciences, Hsinchu, Taiwan where part of this work was done. P.S. acknowledges support of the swedish VR.
1 C.W.J. Beenakker and H. van Houten, Solid State Physics 44, 1 (1991).

${ }^{2}$ Y. Ji, Y. Chung, D. Sprinzak, M. Heilblum, D. Mahalu, and H. Shtrikman, Nature 422, 415 (2003).

3 I. Neder, M. Heiblum, Y. Levinson, D. Mahalu, and V. Umansky, Phys. Rev. Lett. 96, 016804 (2006). 
${ }^{4}$ L. V. Litvin, H.-P. Tranitz, W. Wegscheider, and C. Strunk, cond-mat/0607758

${ }^{5}$ G. Seelig and M. Büttiker, Phys. Rev. B 64, 245313 (2001).

${ }^{6}$ F. Marquardt and C. Bruder, Phys. Rev. Lett. 92, 56805 (2004).

7 F. Marquardt and C. Bruder, Phys. Rev. B 70, 125305 (2004).

8 H. Förster, S. Pilgram, and M. Büttiker, Phys. Rev. B 72, 075301 (2005).

${ }^{9}$ F. Marquardt, Europhys. Lett. 72, 788 (2005).

10 V. S.-W. Chung, P. Samuelsson, and M. Büttiker, Phys. Rev. B 72, 125320 (2005).

11 S. Pilgram, P. Samuelsson, H. Förster, and M. Büttiker, Phys. Rev. Lett. 97, 066801 (2006).

12 H. Förster, P. Samuelsson, S. Pilgram, and M. Büttiker, cond-mat/0609544

13 E.V. Sukhorukov and V.V. Cheianov, cond-mat/0609288

14 D. J. Thouless, Phys. Rev. B 27, 6083 (1983).

15 M. Büttiker, H. Thomas, and A. Pretre, Z. Phys. B: Condens. Matter 94, 133 (1994).

16 B. Spivak, F. Zhou, and M. T. Beal Monod, Phys. Rev. B 51, 13226 (1995).

17 P. W. Brouwer, Phys. Rev. B 58, R10135 (1998).

18 F. Zhou, B. Spivak, and B. Altshuler, Phys. Rev. Lett. 82, 608 (1999).

19 T. A. Shutenko, I. L. Aleiner, and B. L. Altshuler Phys. Rev. B 61, 10366 (2000).

20 J. E. Avron, A. Elgart, G. M. Graf, and L. Sadun, Phys. Rev. B 62, R10618 (2000).

21 M. G. Vavilov, V. Ambegaokar, and I. L. Aleiner, Phys. Rev. B 63, 195313 (2001)

${ }^{22}$ B. Wang, J. Wang, and H. Guo, Phys. Rev. B 65, 073306 (2002).

23 M. Moskalets and M. Büttiker, Phys. Rev. B. 66, 035306 (2002).

24 M. L. Polianski and P. W. Brouwer, J. Phys. A: Math. Gen. 36, 3215 (2003).

25 J. E. Avron, A. Elgart, G. M. Graf, and L. Sadun, J. Stat. Phys. 116, 425 (2004).

${ }^{26}$ M. Martinez-Mares, C. H. Lewenkopf, and E. R. Mucciolo, Phys. Rev. B. 69, 085301 (2004).

27 H.-Q. Zhou, U. Lundin, S. Y. Cho, and R. H. McKenzie, Phys. Rev. B 69, 113308 (2004).

${ }^{28}$ D. Shin and J. Hong, Phys. Rev. B. 70, 073301 (2004).

29 S.W. Chung, C.S. Tang, C.S. Chu, and C.Y. Chang, Phys. Rev. B 70, 085315 (2004).

30 M. Governale, F. Taddei, R. Fazio and F.W.J. Hekking, Phys. Rev. Lett. 95, 256801 (2005).

31 D. Cohen, T. Kottos, and H. Schanz, Phys. Rev. E 71 , 035202(R) (2005).

32 C. Benjamin, Eur. Phys. J. B, 52, 403 (2006).

${ }^{33}$ M. Switkes, C. M. Marcus, K. Campman, and A. C. Gos- sard, Science 283, 1905 (1999).

34 L. DiCarlo, C.M. Marcus, and J.S. Harris, Jr, Phys. Rev. Lett. 91, 246804 (2003).

35 R. Citro and F. Romeo, Phys. Rev. B 73, 233304 (2006).

36 S.K. Kim, K.K. Das, and A. Mizel, cond-mat/0609601.

37 P. Samuelsson and M. Büttiker, Phys. Rev. B 71, 245317 (2005)

38 M. Moskalets and M. Büttiker, Phys. Rev. B 66, 205320 (2002).

39 M. Moskalets and M. Büttiker, Phys. Rev. B 69, 205316 (2004).

40 S.W. Kim, Phys. Rev. B 66, 235304 (2002).

41 P. W. Brouwer, Phys. Rev. B 63, 121303(R) (2001).

42 M. Büttiker, Phys. Rev. B, 46, 12485 (1992).

${ }^{43}$ M. Moskalets and M. Büttiker, Phys. Rev. B 72, 035324 (2005)

44 H. A. Fertig, Phys. Rev. B 38, 996 (1988).

45 M. Büttiker and M. Moskalets, Lecture Notes in Physics 690, 33 (2006).

46 T. Christen and M. Büttiker, Europhys. Lett. 35, 523 (1996).

47 M. H. Pedersen and M. Büttiker, Phys. Rev. B 58, 12993 (1998).

48 M. Büttiker, Phys. Rev. B 33, 3020 (1986).

49 M. Büttiker, IBM J. Res. Dev. 32, 63 (1988).

50 Ya. M. Blanter and M. Büttiker, Phys. Rep. 336, 1 (2000).

51 C. Texier and M. Büttiker, Phys. Rev. B 62, 7454 (2000).

52 S. Oberholzer, E. Bieri, C. Schönenberger, M. Giovannini, and J. Faist, Phys. Rev. Lett. 96, 46804 (2006).

53 M. Moskalets and M. Büttiker, Phys. Rev. B 64, 201305(R) (2001).

54 J.N.H.J. Cremers and P.W. Brouwer, Phys. Rev. B 65, 115333 (2002).

55 M. L. Polianski, P. Samuelsson, and M. Büttiker, Phys. Rev. B 72, 161302(R) (2005).

56 M.L. Polianski, and P.W. Brouwer, Phys. Rev. B 64 075304 (2001).

57 P. Samuelsson, E.V. Sukhorukov and M. Büttiker, Phys. Rev. Lett. 92, 026805 (2004).

58 C.W.J. Beenakker, C. Emary, M. Kindermann, and J.L. van Velsen, Phys. Rev. Lett. 91, 147901 (2003).

59 T. M. Stace, C. H. W. Barnes, and G. J. Milburn, Phys. Rev. Lett. 93, 126804 (2004).

60 C.W.J. Beenakker, M. Titov, and B. Trauzettel, Phys. Rev. Lett. 94, 186804 (2005).

${ }^{61}$ K. K. Das, S. Kim, and A. Mizel, Phys. Rev. Lett. 97, 096602 (2006).

62 A. V. Lebedev, G. B. Lesovik, and G. Blatter, Phys. Rev. B 72, 245314 (2005).

63 M. Moskalets and M. Buttiker, Phys. Rev. B 73, 125315 (2006). 